\title{
Letter
}

\section{Stability in higher-derivative matter fields theories}

\author{
Petr V. Tretyakov ${ }^{1,2, a}$ \\ ${ }^{1}$ Bogoliubov Laboratory of Theoretical Physics, Joint Institute for Nuclear Research, Joliot-Curie 6, Dubna 141980, Russia \\ 2 Department of General Relativity and Gravitation, Institute of Physics, Kazan Federal University, Kremlevskaya street 18, Kazan 420008, Russia
}

Received: 19 August 2016 / Accepted: 1 September 2016/ Published online: 12 September 2016

(c) The Author(s) 2016. This article is published with open access at Springerlink.com

\begin{abstract}
We discuss possible instabilities in higherderivative matter field theories. These theories have two free parameters $\beta_{1}$ and $\beta_{4}$. By using a dynamical system approach we explicitly demonstrate that for the stability of Minkowski space in an expanding universe we need the condition $\beta_{4}<0$. By using the quantum field theory approach we also find an additional restriction for the parameters, $\beta_{1}>-\frac{1}{3} \beta_{4}$, which is needed to avoid a tachyon-like instability.
\end{abstract}

\section{Introduction}

The unsolved problems of General Relativity such as dark energy $[1,2]$ and dark matter [3] force us to investigate different alternatives. One of the most popular alternatives is well-known $f(R)$-gravity [4,5] in the different forms [6-8]. There are also a number of another possibilities to modify gravity, such as Palatini $f(R)$-gravity [9], teleparallel gravity [10], Horndeski theory [11], theories with non-minimal kinetic coupling [12], and so on [13].

A new type of modified gravity was recently proposed [14]. The higher-derivative matter fields are implied in such a kind of theory, which can be interpreted as non-dynamical auxiliary fields,

$G_{\mu \nu}+\Lambda g_{\mu \nu}=T_{\mu \nu}+S_{\mu \nu}(\mathbf{g}, \mathbf{T})$.

The most general form of the term $S_{\mu \nu}$ if we take into account terms up to fourth order in derivatives is

$$
\begin{aligned}
S_{\mu \nu}= & \alpha_{1} g_{\mu \nu} T+\alpha_{2} g_{\mu \nu} T^{2}+\alpha_{3} T T_{\mu \nu}+\alpha_{4} g_{\mu \nu} T^{\alpha \beta} T_{\alpha \beta} \\
& +\alpha_{5} T^{\alpha}{ }_{\mu} T_{\alpha \nu}+\beta_{1} \nabla_{\mu} \nabla_{\nu} T+\beta_{2} g_{\mu \nu} \square T+\beta_{3} \square T_{\mu \nu} \\
& +2 \beta_{4} \nabla^{\alpha} \nabla_{(\mu} T_{\nu) \alpha}
\end{aligned}
$$

where since we impose the divergence free condition $S_{; \mu}^{\mu \nu}=$ $0^{1}$ there are a number of relations between the coefficients. So finally we have only two independent parameters, $\beta_{1}$ and $\beta_{4}$, and Eq. (1) takes the form [14]

$$
\begin{aligned}
G_{\mu \nu}= & T_{\mu \nu}-\Lambda g_{\mu \nu}-\beta_{1} \Lambda g_{\mu \nu} T+\frac{1}{2} \beta_{4} g_{\mu \nu} T^{\alpha \beta} T_{\alpha \beta} \\
& +\frac{1}{4}\left(1-2 \beta_{1} \Lambda\right)\left(\beta_{1}-\beta_{4}\right) g_{\mu \nu} T^{2} \\
& +\left[\beta_{4}\left(1-2 \beta_{1} \Lambda\right)-\beta_{1}\right] T T_{\mu \nu}-2 \beta_{4} T^{\alpha}{ }_{\mu} T_{\alpha \nu} \\
& +\beta_{1} \nabla_{\mu} \nabla_{\nu} T-\beta_{1} g_{\mu \nu} \square T-\beta_{4} \square T_{\mu \nu} \\
& +2 \beta_{4} \nabla^{\alpha} \nabla_{(\mu} T_{\nu) \alpha} .
\end{aligned}
$$

Some of the terms from Eq. (3) also appear in $f(R, T)$ theory [15]. In this sense there may a further generalizations of such a kind of theories, for instance, in the light of $f\left(R, T, R_{\mu \nu} T^{\mu \nu}\right)$-theory [16] or maybe $f\left(R, T, R_{\mu \nu} T^{\mu \nu}\right.$, $\left.T_{\mu \nu} T^{\mu \nu}\right)$-theory. Note that for a special choice of parameters some of the well-known theories are contained in the representation (3) as a limit. For instance, the case $\beta_{1}=0$, $\beta_{4}=-\kappa / 2$ corresponds to EiBI gravity in the small coupling limit, or $\beta_{4}=0$ corresponds to generic Palatini $f(R)$ gravity [14]. In this sense the theory of (1) also may be interpreted as some kind of phenomenological theory of modified gravity. Cosmology in such a kind of theories was investigated in $[17,18]$. There was obtained an effective dark energy sector and one found accelerating, de Sitter, and non-accelerating solutions. Also it was demonstrated that in such a kind of theories the effective dark energy e.o.s. may be quintessencelike, cosmological constant-like or even phantom-like. Nevertheless we need to note that the problem of multiple de Sitter solutions (which was successfully solved in $f(R)$ gravity [19]) is unsolved yet, and it seems that it cannot be solved without some additional terms in the equation. Generalizations for brane theories were studied in [20-22]. Some interesting remarks about this theory may be found also in [23].

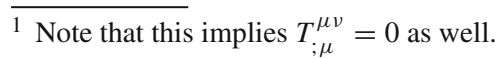


For a standard perfect fluid $T_{\mu \nu}=(p+\rho) U_{\mu} U_{v}+p g_{\mu \nu}$ and FLRW metric $\mathrm{d} s^{2}=-\mathrm{d} t^{2}+a^{2}(t) \delta_{\mu \nu} \mathrm{d} x^{\mu} \mathrm{d} x^{\nu}$ the Friedman-like equation takes the form [17]

$$
\begin{aligned}
3 H^{2}= & \rho+\Lambda+3 H\left[\beta_{1}(\dot{\rho}-3 \dot{p})-\beta_{4}(\dot{\rho}+2 \dot{p})\right]-\beta_{4} \ddot{\rho} \\
& +\frac{1}{4}\left[3 \beta_{1}\left(1+2 \beta_{4} \Lambda\right)\left(\rho^{2}-3 p^{2}\right)\right. \\
& +3 \beta_{4}\left(\rho^{2}+p^{2}\right)-12 \beta_{1}\left(\beta_{1}+\beta_{4}\right) \Lambda \rho p \\
& -6\left(\beta_{1}-\beta_{4}\right) \rho p-4 \beta_{1} \Lambda(\rho-3 p) \\
& \left.+2 \beta_{1}^{2} \Lambda\left(\rho^{2}+9 p^{2}\right)\right],
\end{aligned}
$$

and also we have the usual energy conservation law $T_{; \mu}^{\mu \nu}=0$, which reads now

$\dot{\rho}+3 H(p+\rho)=0$,

where $H=\frac{\dot{a}}{a}$ is usual Hubble parameter.

\section{Stability conditions}

It is well known that incorporating of higher-derivative terms can affect the stability of different solutions including the simplest cosmologically relevant one. One of the most important is the Minkowski solution. Let us study the stability of the Minkowski solution in such a kind of gravity. To discuss the stability problem we need to transform the equations to the form of a dynamical system. First of all we will discuss the simplest (and for cosmological applications the most convenient) equation of state, $p=w \rho$. Further we can express the Hubble parameter from Eq. (5) and insert it into (4). In this way we obtain a second order differential equation for the function $\rho$, which is also dependent on the parameters $\beta_{1}, \beta_{4}, \Lambda$, and $w$ :

$\beta_{4} \ddot{\rho}=\rho+\Lambda+\frac{1}{4} \rho^{2} f_{2}-\frac{f_{1}}{1+w} \frac{\dot{\rho}^{2}}{\rho}-\frac{1}{3(1+w)^{2}} \frac{\dot{\rho}^{2}}{\rho^{2}}$,

with

$f_{1}=\beta_{1}(1-3 w)-\beta_{4}(1+2 w)$,

and

$$
\begin{aligned}
f_{2}= & 3 \beta_{1}\left(1-3 w^{2}\right)\left(1+2 \beta_{4} \Lambda\right)+3 \beta_{4}\left(1+w^{2}\right) \\
& -12 \beta_{1}\left(\beta_{1}+\beta_{4}\right) \Lambda w-6 w\left(\beta_{1}-\beta_{4}\right) \\
& -4 \beta_{1}(1-3 w) \Lambda+2 \beta_{1} \Lambda\left(1+9 w^{2}\right) .
\end{aligned}
$$

We can see that $f_{1}$ and $f_{2}$ are non-dynamical functions and they only depend on the parameters of the theory.

For further investigation let us rewrite Eq. (6) as a dynamical system:

$$
\left\{\begin{array}{c}
\dot{\rho}=\pi, \\
\dot{\pi}=F,
\end{array}\right.
$$

with the function $F$

$$
\begin{aligned}
F(\rho, \pi) \equiv & \frac{1}{\beta_{4}}\left(\rho+\Lambda+\frac{1}{4} \rho^{2} f_{2}-\frac{f_{1}}{1+w} \frac{\pi^{2}}{\rho}\right. \\
& \left.-\frac{1}{3(1+w)^{2}} \frac{\pi^{2}}{\rho^{2}}\right) .
\end{aligned}
$$

It is clear that the stationary points of system (9) are governed by the next simple equation,

$\frac{1}{4} f_{2} \rho^{2}+\rho+\Lambda=0$.

Now let us discuss the physical meaning of its solutions. First of all, note that we are interested in solutions with a true vacuum $\Lambda=0$, but we need to keep the parameter $\Lambda$ to investigate perturbations because it is coupled with the other parameters of our theory, $\beta_{1}$ and $\beta_{4}$. So we can let it vanish it only at the end of our investigation. Therefore, we may interpret $\Lambda$ as a parameter which allows us to avoid degeneration of the solutions and we can put $\Lambda \rightarrow 0$ at the end. A similar approach was successfully practiced to a stability investigation of the Minkowski solution in different modified gravity theories in our previous papers [24,25]. The first solution of Eq. (11) reads

$\rho=\frac{-2-2 \sqrt{1-f_{2} \Lambda}}{f_{2}}$

We can see that in the case $\Lambda \rightarrow 0$ this solution reads $\rho=-\frac{4}{f_{2}} \neq 0$; moreover, (5) tells us that $H=0$. So this solution corresponds to the static Einstein universe and it is not interesting for our further investigations; but if we want to keep it, we must put $f_{2}<0$ to satisfy the weak energy condition (wec) $)^{2}$ and some additional restrictions for the parameters will follow from this inequality. ${ }^{3}$ The second solution reads

$\rho=\frac{-2+2 \sqrt{1-f_{2} \Lambda}}{f_{2}}$

and it has the limit $\rho \rightarrow-\Lambda$ for $\Lambda \rightarrow 0$, so we need to put $\Lambda \rightarrow-0$ to satisfy wec. Note that this solution exists for any value of $H$ including the case $H=0$. In the last case this is a Minkowski solution and its stability is very important for us. Let us study the Minkowski stability conditions. It is well known that in the first order, stability is governed by the equation

$\left|\begin{array}{cc}-\mu & 1 \\ \left(F_{\rho}\right)_{0} & \left(F_{\pi}\right)_{0}-\mu\end{array}\right|=0$,

\footnotetext{
${ }^{2}$ Note that in our case $p=w \rho, w \neq-1$ and wec directly follows from the null energy condition (nec).

${ }^{3}$ It reads $\beta_{1}<\frac{\beta_{4}(w+1)}{3 w-1}$ or for the dust $(w=0)$ case $\beta_{1}<-\beta_{4}$.
} 
or $^{4}$

$\mu^{2}-\left(F_{\pi}\right)_{0} \mu-\left(F_{\rho}\right)_{0}=0$,

and it easy to see that the stability conditions take the form ${ }^{5}$

$\left\{\begin{array}{l}\left(F_{\pi}\right)_{0}<0, \\ \left(F_{\rho}\right)_{0}<0 .\end{array}\right.$

Let us calculate the values of these functions explicitly. For the function $F_{\pi}$ we have

$F_{\pi}=\frac{-2 \dot{\rho}}{(1+w) \rho} \frac{1}{\beta_{4}}\left[f_{1}+\frac{1}{3(1+w) \rho}\right]$.

First of all note that at the interesting point this function takes a singular value, but we need only its sign. Second, we can put $1+w>0$ without loss of generality, because the case $w=-1$ corresponds to the case of the cosmological term, which is already incorporated in our equations. Now we can see from (5) that $\frac{-2 \dot{\rho}}{(1+w) \rho}=6 H>0$ in an expanding universe. The value of the function $f_{1}$ is always finite, see (7), whereas the value of the expression $\frac{1}{3(1+w) \rho}$ is infinite at the point we study $(\rho=0)$, and positive if we pose the quite natural conditions $1+w>0$ and $\rho>0$. So we find that the total sign of Eq. $(17)$ at the point $(0,0)$ is governed by the sign of the parameter $\beta_{4}$ and for Minkowski stability we must have $\beta_{4}<0$.

Now let us discuss the second condition from (16). We have

$F_{\rho}=\frac{1}{\beta_{4}}+\frac{1}{2} \rho f_{2}+\frac{f_{1}}{\beta_{4}(1+w)} \frac{\dot{\rho}^{2}}{\rho^{2}}+\frac{2}{3 \beta_{4}(1+w)^{2}} \frac{\dot{\rho}^{2}}{\rho^{3}}$.

First of all note that near the interesting point $(0,0)$ the second term may be neglected because $f_{2}$ always has a finite value, see Eq. (8), and $\rho \rightarrow 0$, whereas the first term is equal to a non-zero constant. According to (5) $\frac{\dot{\rho}^{2}}{\rho^{2}}=$ $9 H^{2}(1+w)^{2} \rightarrow 0$ for the Minkowski solution, so the third term may also be neglected. And for the fourth term we have $\frac{\dot{\rho}^{2}}{\rho^{3}(1+w)^{2}}=\frac{9 H^{2}}{\rho}=3$ near the point $\rho=0$; see (4). So finally we have

$\left(F_{\rho}\right)_{0}=\frac{3}{\beta_{4}}$,

and we can see that for stability we need $\beta_{4}<0$ as well.

Now let us discuss possible instabilities from another point of view. The trace of Eq. (3) reads

$$
\left(\beta_{4}+3 \beta_{1}\right) \square T+2 \Lambda\left(2+2 \beta_{1} T+\beta_{1}^{2} T^{2}\right)-T-R=0 .
$$

\footnotetext{
$\overline{{ }^{4} \text { Here we imply }} F_{\rho} \equiv \frac{\partial F}{\partial \rho}$ and so on.

${ }^{5}$ Recall that $\operatorname{Re}\left(\mu_{1,2}\right)<0$ is needed for stability.
}

Let us study a small perturbation $\delta T$ for some solution of this equation with $T_{0}$ and $R_{0}$. So we put $T=T_{0}+\delta T$ and the equation for $\delta T$ takes the form

$\left(\beta_{4}+3 \beta_{1}\right) \square \delta T+4 \Lambda\left(\beta_{1}+2 \beta_{1}^{2} T_{0}\right) \delta T-\delta T=0 ;$

we try to find possible solutions of (21) as standard decomposition on the functions

$u_{k} \sim e^{i \mathbf{k} \mathbf{x}-i \omega t}$,

where $\omega \equiv\left(k^{2}+\mu^{2}\right)^{1 / 2}, k \equiv|\mathbf{k}|$, and $\mu$ is the mass of the effective scalar field (scalaron). After substituting this representation into (21) we obtain the next equation for $\mu^{2}$ :

$\mu^{2}=\frac{1-4 \Lambda\left(\beta_{1}+2 \beta_{1}^{2} T_{0}\right)}{\beta_{4}+3 \beta_{1}}$,

and if we turn back to the Minkowski limit $\Lambda \rightarrow 0$, this relation give us an additional restriction to avoid the tachyonlike instability $\left(\mu^{2}<0\right)$ :

$\beta_{1}>-\frac{1}{3} \beta_{4}$.

\section{Conclusion}

In this paper we discuss instabilities in higher-derivative matter field theories. We found the condition for the Minkowski stability and another one to avoid the tachyon-like instability. Of course, these are only necessary but not sufficient conditions for stability of the theory. For instance we study Minkowski stability only with respect to the simplest class of isotropic perturbations and taking into account more complicated perturbations may provide us with some additional restrictions on the parameters. Nevertheless the conditions found must be satisfied and may be very helpful for further construction of the theory.

Acknowledgments The author is grateful to A.A. Starobinsky for discussion and some useful remarks. This work was supported by the Russian Science Foundation (RSF) grant 16-12-10401.

Open Access This article is distributed under the terms of the Creative Commons Attribution 4.0 International License (http://creativecomm ons.org/licenses/by/4.0/), which permits unrestricted use, distribution, and reproduction in any medium, provided you give appropriate credit to the original author(s) and the source, provide a link to the Creative Commons license, and indicate if changes were made.

Funded by $\mathrm{SCOAP}^{3}$.

\section{References}

1. A.G. Riess et al., Astron. J. 116, 1009 (1998)

2. S. Perlmutter et al., Astrophys. J. 517, 565 (1999)

3. P.A.R. Ade et al. (Planck Collaboration), arXiv:1502.01589

4. K. Bamba, S. Capozziello, S. Nojiri, S.D. Odintsov, Astrophys. Space Sci. 342, 155 (2012) 
5. S. Nojiri, S.D. Odintsov, Int. J. Geom. Meth. Mod. Phys. 4, 115 (2007)

6. A.H. Chamseddine, V. Mukhanov, JHEP 1311, 095 (2013)

7. E. Alvarez, JHEP 0503, 002 (2005)

8. S. Nojiri, S.D. Odintsov, V.K. Oikonomou, JCAP 05, 046 (2016)

9. T. Harko, T.S. Koivisto, F.S.N. Lobo, G.J. Olmo, Phys. Rev. D 85, 084016 (2012)

10. R. Aldrovandi, J.G. Pereira, Teleparallel gravity: an introduction (Springer, Dordrecht, 2012)

11. T. Kobayashi, M. Yamaguchi, J. Yokoyama, Prog. Theor. Phys. 126, 511 (2011)

12. S.V. Sushkov, Phys. Rev. D 80, 103505 (2009)

13. T. Clifton, P.G. Ferreira, A. Padilla, C. Skordis, Phys. Rep. 513(1), 1-189 (2012)

14. P. Pani, T.P. Sotiriou, D. Vernieri, Phys. Rev. D 88, 121502 (2013)

15. T. Harko, F.S.N. Lobo, S. Nojiri, S.D. Odintsov, Phys. Rev. D 84, $024020(2011)$

16. S.D. Odintsov, D. Saez-Gomez, Phys. Lett. B 725, 437-444 (2013)
17. T. Harko, F.S.N. Lobo, E.N. Saridakis, Int. J. Geom. Methods Mod. Phys. 13, 1650102 (2016)

18. D. Bazeia, F.A. Brito, F.G. Costa, Phys. Rev. D 90, 043523 (2014)

19. G. Cognola, E. Elizalde, S.D. Odintsov, P. Tretyakov, S. Zerbini, Phys. Rev. D 79, 044001 (2009)

20. B. Guo, Y.-X. Liu, K. Yang, Eur. Phys. J. C 75(2), 63 (2015)

21. D. Bazeia, A.S. Lobao Jr., R. Menezes, Phys. Rev. D 90, 067702 (2014)

22. D. Bazeia, M.A. Marques, R. Menezes, D.C. Moreira, Ann. Phys. 361, 574 (2015)

23. E. Berti et al., Class. Quantum Gravity 32, 243001 (2015)

24. P.V. Tretyakov, Int. J. Geom. Methods Mod. Phys. 12, 1550094 (2015)

25. P.V. Tretyakov, Mod. Phys. Lett. A 31, 1650085 (2016) 